\title{
RePhyS: A Multidisciplinary Experience in Remote Physiological Systems Laboratory
}

\author{
http://dx.doi.org/10.3991/ijoe.v9iS5.2756 \\ C. Barros, C. P. Leão, F. Soares, G. Minas, J. Machado \\ University of Minho, Guimarães, Portugal
}

\begin{abstract}
Remote laboratories have been developed to support the students' learning and to reduce equipment costs. More and more, laboratories play an important role in engineering teaching, since they allow the connection between theory and practice. Actually, there is the necessity to change strategies and methodologies of education. The remote laboratories enable the access to all students anytime, anywhere because they are available on the Internet. This paper presents the firsts developments of an innovative laboratory in the bioengineering area: RePhyS. RePhyS will be a remote laboratory for use in biomedical and physiological systems studies in graduate and postgraduate levels. The user will be allowed to interact with the system parameters, and to control some system variables. This new lab will enable to produce, visualize and analyze, in real-time, results of practical experiences in order to improve students' activity of learning, and it will provide with an enriched computing environment for sharing results and collaborations.
\end{abstract}

Index Terms-bioengineering education; physiological systems; remote and virtual labs.

\section{INTRODUCTION}

The laboratorial experiments play an important role in the sciences and engineering teaching [1]. The students should acquire knowledge and develop their own capacities, requiring the recurrent, or even intensive, use of laboratories where they can combine theory with practice $[1,2]$. The use of laboratories demands time, space and large financial investments to obtain the equipment, which is not always within reach of education institutions $[2,3]$. The necessity of laboratorial practice leads to creation or development of virtual and remote laboratories [2], providing the distance teaching by the use of organized educational resources [4]. The main goal of this kind of laboratories is to offer an interactive platform between the real world and the experimentation, the problems resolution, the achievement and the scientific interpretation of data [1-6].

\section{A. Virtual Labs}

In education, the simulation has been used to illustrate events that are not easily visualized. The simulators are used as assistants or even substitutes of laboratorial experiences. This is possible because they perform mathematical equations of physic phenomena that can be developed and modeled with some accuracy [7]. The virtual instruments provide an amount of practical experiments of real world and they are designed to offer to student the possibility of working with problems of real life that emphasize the theory $[5,6,8]$.
This kind of laboratories presents some advantages such as the obtainment of effective explanations for theoretical concepts; the step by step leading of experiences; the interactive platform; the friendly use; the inexistence of time or space limitations, consequently, a more flexibility; and the lower costs compared with real laboratories' costs [3]. However, they do not allow "hands on" experiences, that is, experiences that need physics experiments and practical work, as in a real laboratory. Therefore they cannot be fully replaced [3, 7]. Furthermore, the practical knowledge and the experience acquired by student depend on the authenticity and the skills of the software. In a real laboratory, the inputs and outputs predesigned are limited, restricting the student's creativity [8].

\section{B. Remote Labs}

A remote laboratory is composed by a group of experimental platforms that allow the interactive access of its resources at distance [4].

The Internet comes to solve the difficulty of providing laboratorial experiments at distance. It is able to interconnect nodes of technology in an almost instantaneous way and with this capability it changes the education at distance, as well as the expectations of both students and teachers $[7,8]$.

This kind of laboratory is a new concept that enables remote users to feel as if they are actually present in the real laboratory at different place or time $[3,9]$. Contrary to virtual simulations on the web, the students have the possibility to directly control the real laboratorial experiments through the remote access to the laboratorial equipment [10]. This is possible through Internet connection, allowing the access anytime and anywhere $[2,4,6,8$, $10]$. The student can adjust the control parameters in the webpage; after that, the parameters are sent through server that controls the experience and executes it in a real laboratory; finally, the results are presented to the student $[2,6,8]$.

The remote control of real experiments is a potential costs reducer, increasing the effectiveness in the equipment's utilization and warranting access to every student $[1,6,10]$. In addition, the remote labs have others advantages such as their flexibility, because of their time and space independency, the possibility of access to a large number of students and to students with physical deficiencies [4]. They also afford the learning by trial and error and it allows the real data analysis, which is relevant to acquire some practical skills. The only disadvantage is the virtual presence of the student [3]. 


\section{Biomedical Labs}

The biomedical engineering is growing as research and development area, as knowledge, as practice in industry and in healthcare. It requires students who adapt easily to the new information and who recognize the potential of its application in the human health [11].

The biomedical engineering laboratories must be designed in order to enable students' interaction with real problems and understanding the acquired theoretical concepts. The students must learn the basics of the acquisition, the processing and the data presentation [5].

The biomedical engineering education offers a range of challenges to all the component parts of the educational process: faculty, students, employers and graduates [11]. The education technologies are the potential to achieve with efficiency didactic environments focused on student, on knowledge and in evaluation [11]. Sometimes the cost of the equipment hinders the integration of acquisition systems of physiological signals in university laboratories [12]. The application of analysis systems is a very important field in biomedical engineering studies because they are able to model, stimulate and control the most important physiological systems [13]. The virtual laboratories can be built in an interactive way, and students can qualitatively understanding the behavior of complex models used to represent the human body [13].

\section{Motivation}

\section{A. A brief literature review}

The development of new learning technologies increases the pedagogical process effectiveness [11]. Several virtual and remote laboratories have been proposed in scientific and technical educational areas [14]. iLabs is a project which is dedicated to the online laboratories, real laboratories accessed through the Internet, with interest in areas such as biology, chemistry or physics [15]. The WebLab is one of the laboratories developed under the iLabs project and make microelectronics test equipment [16]. Others virtual and remote laboratories already exist to support the engineering students' learning $[6,17,18]$. As described in [6] a web assisted laboratory for on-line control engineering was designed allowing students to access several virtual and remote laboratories experiences on automation, process control and numerical methods. The MicroLab is a good example of a web-based multiuser remote microcontroller laboratory designed for electric-electronic engineering education [17]. The Remote Lab [18] is another example and it is also operating in the area of engineering education. The main purpose of this remote laboratory is to study mechanical material characteristics [19].

Few remote labs works founded on the web are focused on physiological systems experiments [20] besides virtual laboratories for the same kind of experiences are very common. For instance, Biotechnology and biomedical engineering [21], Neuron Simulation Virtual Lab [22] or biomedical Instrumentation are laboratories developed or in development under Virtual Labs project [23]. This present proposal intends to overcome this gap. In this research the design and implementation of middleware architecture, sufficiently flexible and expandable to produce different experiments with the remote access to physical devices connected to the Internet, will give to the student a realistic perception of the real physical experiment.

\section{B. RePhyS}

The application of analysis systems is a very important field in biomedical engineering studies because they are able to model, simulate and control the most important physiological systems [20]. The RePhyS (Remote Physiological Systems) laboratory is the remote lab focused on physiological systems research. It will be an innovative remote laboratory for biomedical and physiological systems studies. It should include different real experiments with the analogues of biomedical systems, remotely visualized by a webcam; it must be modular and dynamic, accepting new practical problems; it should include the theoretical support of the practical problems; it should include the principles of a social network, being the support to the students learning process through the communication and sharing of resources.

\section{METHODS AND PLANS}

The initial phase of remote lab development is being done using a kit for physiological signals measurements: the Bio Starter Kit ${ }^{\circledR}$ from Shimmer Research ${ }^{\mathrm{TM}}$. This kit is composed by four sensor modules for the acquisition of ECG (electrocardiogram), EMG (electromyography), GSR (galvanic skin response) and Strain Gauge [24]. The Shimmer ${ }^{\mathrm{TM}}$ unit provides data capture, processing, storage and transmission to a computer by Bluetooth connection (Figure 1). It also includes development software tools for wireless solutions, using LabVIEW applications.

The use of biomedical equipment through remote laboratory will be made by means of two applications. The first one refers to the physiological signal acquisition from human body, and the second one using mechanical simulators of biomedical systems.

Initially, the physiological signals that may be able to be acquired will be restricted to the sensor modules of ShimmerTM device, always requiring a subject to undergo the experience in the real laboratory.

The development of biomechanical simulators of biological systems is also part of this project. The cardiovascular, respiratory and muscular systems are already in developing. This task aims the construction of a physical model and the application of electronics, mechanics and other engineering concepts to simulate those systems.

With these possibilities, the research team is developing a web interface for measuring, recording and processing the various signals acquired remotely from both human body and physiological systems simulators.

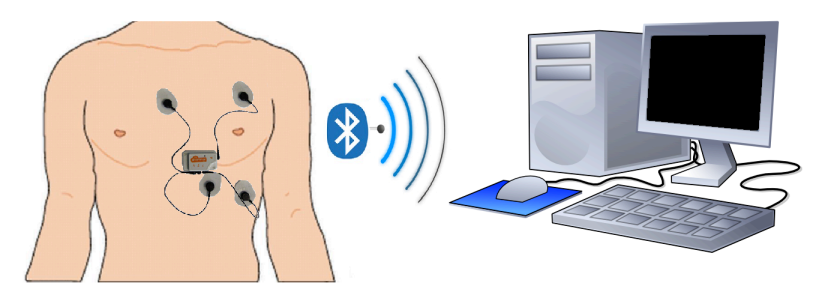

Figure 1. Scheme of local acquisition and transmission of physiological data using Shimmer ${ }^{\mathrm{TM}}$ device (adapted from [24]). 


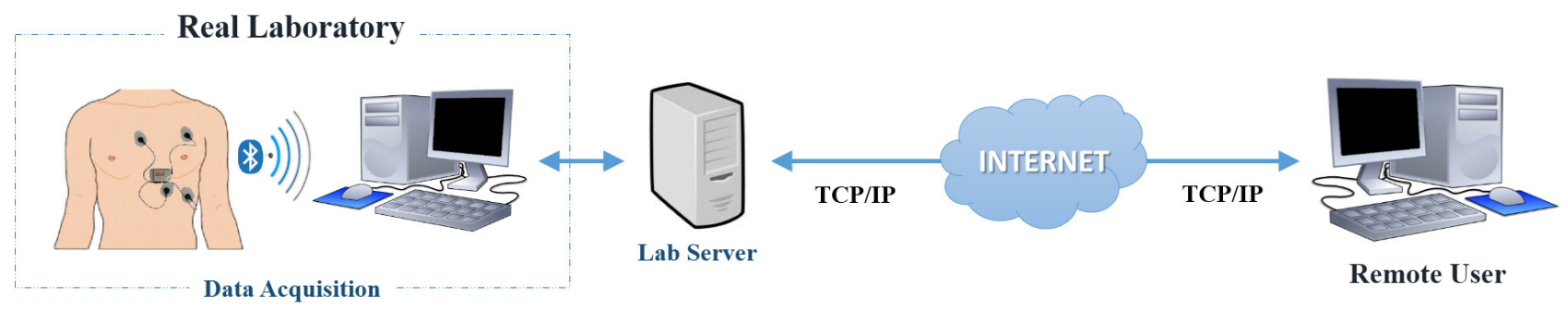

Figure 2. Proposal general architecture for the remote lab RePhyS.

The development of a remote lab includes the analysis of user requirements, including, among others, remote control functionalities, simultaneous user operation sharing the online data from an experiment, read data, change variables, full control, and time constrained user interactions. Functional requirements are associated with specific functions or services that the system must support.

The LabVIEW is the software used to assist the development of web services and applications, which will control the processes. Indeed, this software owns a webserver allowing the experiences' publication via Internet. LabVIEW application will be connected to the experience and it will allow the web service generation. This web service will use the Internet protocols and norms for an immediate interaction with the user, by a web browser $[25,26]$. An efficient communication system is required for the coordination of the interaction between the server and the user. The TCP/IP is a robust Internet protocol that ensures the data transmission over the network [20]. The webserver sends the user commands to the lab server in the form of messages through TCP/IP protocol. The lab server hosts the control software and enables commands sending to the device under control, to which is connected, with regard to the received requests from the user [27]. The LabVIEW siftware has the Web Publishing Tool that makes possible the easy web deployment of a front panel, allowing the remote control of this panel, and consequently the remote control of the device, for example.

The proposal architecture for the Remote Lab is presented in Figure 2. With this proposal architecture, the students must be able to send commands through web browser, which could be pre-processed by themselves. These commands will be received by lab server, and then will be executed the experience in the real device. The results will appear on user's interface. With the existence of a webcam on laboratory will become possible driving the experience and visualize it in real-time on browser. The access to the remote lab will be made through sessions: students will initialize the user session before and will end it after the experience, which will determine the user connection to the server [28]. At distance, the remote lab will be accessible by two distinct ways. One, where each experience is ready and the user is able to see all the system parameters, to see the system working (using a webcam), and to see the obtained results fully processed. On the other way to access this remote lab, the user will be allowed to interact with the system parameters and get a display of the results, or the results themselves, for further processing with user own tools. It will be also possible to control some variables.

\section{EDUCATIONAL GOALS}

This project follows the trends in engineering education as a multi and inter disciplinary task. It covers several subjects in different levels of graduation.

The remote lab will provide a platform that should include different real experiments: human body and biomechanical simulators. This lab will aggregate both virtual and remote experiences. The remote experiences define a complementing learning paradigm centered in student interaction and experimentation, where users can produce, visualize and analyze, in real-time, results of practical experiences. The interaction with the measurement's parameters, and the possibility to control them, implies the improvement of student's learning on data acquisition and processing. This practical experimentation coupled with virtual appliances will help students on comprehension of physiological theoretical concepts, as well as the improvement of the bioinstrumentation achievements.

The team's objective is then to improve students' activity of learning, which is very important to knowledge, skill and competency development.

\section{FINAL CONSIDERATIONS}

The RePhyS is a multidisciplinary project which covers a range of knowledge areas: understanding and definition of practical cases in biomedical engineering, process moedeling of physiological systems, numerical simulation, distributed architectures, electronics and mechanical engineering.

With the development and implementation of RePhyS, the research team expects to have a user-friendly interface for the support of students' learning process of physiological systems and to have the Laboratory running online and used as a relevant complementary learning tool for biomedical education.

\section{ACKNOWLEDGMENT}

The authors are grateful to the Portuguese Foundation for Science and Technology (FCT) for funding through the R\&D project PTDC/CPE-PEC/122329/2010.

\section{REFERENCES}

[1] A. C. Ammari, and J. B. H. Slama, The Development of a Remote Laboratory for Internet-Based engineering Education. Journal of Asynchronous Learning Networks 10(4): 3-13, December 2006

[2] A. J. Álvares and J. C. E. Ferreira, Metodologia para Implantação de Laboratórios Remotos Via Internet na Área de Automação da Manufatura, Anais do $2^{\circ}$ Congresso Brasileiro de Engenharia de Fabricação (COBEF) Uberlândia, 2003 (in portuguese). 
[3] Z. Nedic, J. Machotka and A. Nafalski, Remote Laboratories versus Virtual and Real Laboratories, 33rd ASEE/IEEE Frontiers in Education Conference, Boulder, CO, November 5-8, 2003.

[4] A. C. Gasparetti, Laboratório remoto para ensino de Engenharia, Revista Integração, Universidade São Judas Tadeu. São Paulo.

[5] R. D. Trumbower and J. D. Enderle, Virtual instruments in undergraduate biomedical engineering laboratories, IEEE Eng Med Biol Mag. 2003 Jul-Aug; 22(4): 101-10. http://dx.doi.org/10.1109/MEMB.2003.1237509

[6] C. P. Leão, F. Soares, H. Rodrigues, E. Seabra, J. Machado, P. Farinha and S. Costa, Web-Assisted Laboratory for Control Education: Remote and Virtual Environments, Communications in Computer and information Science 282, Uckelmann, D. et al. Eds., 62-72, 2012, Springer. ISBN 978-3-642-28815-9. http://dx.doi.org/10.1007/978-3-642-28816-6

[7] D. F. Lyle and J. R. Albert, The role of the laboratory in undergraduate engineering education, Journal of engineering Education, 2005, 94, 121-130.

[8] B. Balamuralithara and P. C. Woods (2009), Virtual laboratories in engineering education: The simulation lab and remote lab. Comput. Appl. Eng. Educ., 17: 108-118. http://dx.doi.org/ 10.1002/cae.20186

[9] S. S. Fisher, Virtual Environments: Personal Simulations \& Telepresence. In Virtual Reality: Theory, Practice and Promise, S. Helsel and J.Roth, ed., Meckler Publishing, 1991.

[10] D. Popescu and B. Odber, The Advantages of Remote Labs In engineering Education, Educator's Corner, Agilent Technologies, http://cp.literature.agilent.com/litweb/pdf/5990-7694EN.pdf (accessed in September 2012).

[11] T. R. Harris, J. D. Bransford and S. P. Brophy, Roles for learning sciences and learning technologies in biomedical engineering education: A Review of Recent Advances, Annu. Rev. Biomed. Eng. 2002. 4:29-48. http://dx.doi.org/10.1146/annurev.bioeng.4. $\underline{091701.125502}$

[12] B. Land, B. R. Johnson, B. Wyttenbach and R. R. Hoy, Tools for physiology labs: Inexpensive equipment for physiological stimulation, Journal of Undergraduate Neuroscience Education, 2004, vol. 3: A30-A35.

[13] V. Hernandez, M. Alher et al., Virtual laboratory for simulation and learning of cardiovascular system function in biomedical engineering studies. Rev. Fac. Ing. Univ. Antioquia [online]. 2011, n.60, pp. 194-201. ISSN 0120-6230

[14] H. Benmohamed, A. Leleve and P. Prévot, Remote laboratories: new technology and standard based architecture, Proceedings of 2004 International Conference on Information and Communication Technologies: From Theory to Applications (19/04/2004), 101-102.

[15] iLabs Central, Real Labs. Real Learning. - http://ilabcentral.org (accessed in March 2013).

[16] MIT Microelectronics WebLab - http://weblab2.mit.edu (accessed in March 2013).

[17] A. Kutlu, MicroLab: A Web-based Multi-user Remote Microcontroller Laboratory for engineering Education, International Journal of engineering Education, 2004, VOL 20; NUMB 5, 879-885.

[18] Online Lab FEUP - http://remotelab.fe.up.pt (accessed in April 2013).

[19] M. T. Restivo, J. Mendes, A. M. Lopes, C. M. Silva and F. Chouzal, A Remote Laboratory in engineering Measurement, IEEE Transactions on Industrial Electronics, vol.56, no.12, 48364843, Dec. 2009. http://dx.doi.org/10.1109/TIE.2008.2011479
[20] N. S. Shah, (2006) Implementation of Interactive Remote Physiologic Monitoring and Feedback Training System. Master Of Science Thesis, 103 pp. (accessed in March 2013).

[21] Sakshat Virtual Labs - http://coep.vlab.co.in (accessed in February 2013).

[22] Amrita Virtual Labs - http://amrita.vlab.co.in (accessed in February 2013).

[23] Virtual Labs: An Initiative of Ministry of Human Resource Development (MHRD) Under the National Mission on Education through ICT. - http://www.vlab.co.in (accessed in March 2013).

[24] Shimmer: Discovery in Motion - http://www.shimmerreasearch.com (accessed in April 2013).

[25] M. Ngolo, "Arquitetura Orientada a Serviços REST para Laboratórios Remotos", Master Degree Dissertation, Universidade Nova de Lisboa, July 2009 (in portuguese).

[26] J. Djordjevic-Kozarov and M. Arsic, Virtual Laboratory Architecture and Application for Remote Access, TELSIKS 2009, Serbia, 2009.

[27] M. Tawfik, E. S. Cristobal, A. Pesquera, R. Gil, S. Martin and G. Diaz, "Shareable educational architectures for remote laboratories," in Technologies Applied to Electronics Teaching (TAEE), 2012, 2012, pp. 122-127.

[28] S.H. Chen, R. Chen, V. Ramakrishnan, S.Y. Hu, Y. Zhuang, C.C. Ko and Ben M. Chen, Development of Remote Laboratory Experimentation through Internet, Singapore.

\section{AUTHORS}

C. Barros is with the Department of Production and Systems, School of Engineering of University of Minho, Centro ALGORITMI, Guimarães, Portugal (carla.barros@dps.uminho.pt).

C. P. Leão, is with the Department of Production and Systems, School of Engineering of University of Minho, Centro ALGORITMI, Guimarães, Portugal (cpl@dps.uminho.pt).

F. Soares is with the Industrial Electronics Department, School of Engineering of University of Minho, Centro ALGORITMI, Guimarães, Portugal (fsoares@dei.uminho.pt).

G. Minas is with the Industrial Electronics Department, School of Engineering of University of Minho, Centro ALGORITMI, Guimarães, Portugal (gminas@dei.uminho.pt).

J. Machado is with the Mechanical engineering Department, School of Engineering of University of Minho, Mechanical \& Materials Technologies Centre (CT2M), Guimarães, Portugal (jmachado@dem.uminho.pt).

This work is funded by national founds through FCT - Fundação para a Ciência e Tecnologia, under project PTDC/CPE-PEC/122329/2010. This article is an extended and modified version of a paper presented at the EDUCON2013 conference held at Technische Universität Berlin, Berlin, Germany from March 13-15, 2013. Received 09 May 2013. Published as resubmitted by the authors 27 May 2013. 